\title{
PERFORMANCE OF THE CERN SPS FAST EXTRACTION FOR THE CNGS FACILITY
}

\author{
E. Gaxiola, G. Arduini, W. Höfle, F. Roncarolo, E. Vogel, E.Vossenberg \\ CERN, Geneva, Switzerland
}

\section{Abstract}

The SPS LSS4 fast extraction system will serve both the anti-clockwise ring of the LHC and the long baseline neutrino (CNGS) facility. For the latter two extractions spaced by $50 \mathrm{~ms}$, each affecting half of the SPS, are foreseen. During the shutdown 2003-2004 the performance of the fast extraction kickers has been improved in order to match more closely the specifications required for the CNGS and LHC extractions. The kick rise and fall times were significantly reduced, as well as the post-pulse kick ripple. However, the latter remained outside specifications and oscillations were induced in the leading bunches of the batch remaining in the machine at the moment of the first extraction. While further improving the kicker pulse shape, the possibility of damping the beam oscillations using the transverse feedback system has been explored. Recent pulse improvements and results of beam tests are reported.

\section{INTRODUCTION}

The MKE fast extraction kicker system which was used since the late 70's until 2000 in the Super Proton Synchrotron (SPS) has been modernised, upgraded and re-installed in the Long Straight Section 4 (LSS4) to meet the LHC and particularly the CNGS requirements [1-3].

It is a matched travelling wave system comprising five generators and magnets. The pulse forming networks

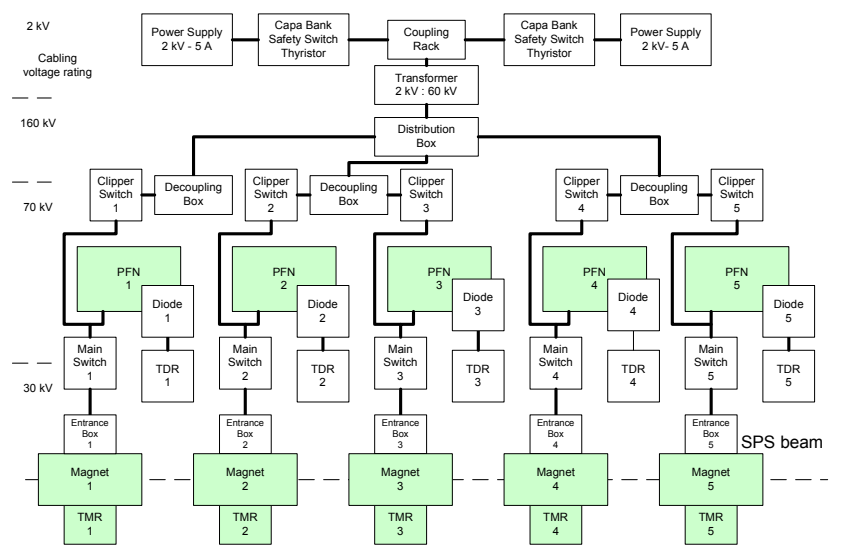

Figure 1: Schematic of the SPS LSS4/ECA4 extraction kicker system (modified components in SD 2003/4 are indicated by a filled pattern).
(PFNs) and magnets consist of lumped LC ladder networks as an approximation to an ideal travelling wave impedance $Z_{0}=\sqrt{L} / C$. They are powered by a resonant charging circuit (see Fig. 1).

As from 2006 one double batch extraction per SPS cycle is foreseen for CNGS. The rising and falling edges of the extraction kick field have to be shorter than the gaps between the two batches, in order to extract the first batch without influencing the one still circulating. Tight constraints have also to be met concerning the flattop ripple which influences the angle under which the various bunches hit the target, located $590 \mathrm{~m}$ downstream of the extraction.

The re-installed MKE system was first tested with beam in 2003, showing that the overall requirements were not yet met in the initial setup. Bunches of the second batch received residual kicks from the first extraction, leading to transverse beam oscillations. This effect was simulated in detail [4]. In the shutdown 2003/2004 several hardware improvements were implemented and the system re-tested with beam in 2004 .

\section{SIMULATIONS AND MEASUREMENTS}

\section{PSpice Simulations}

The kicker system was modelled using PSpice. An evaluation of the effect of varying different parameters showed that magnet damping was necessary to comply with the flattop requirements. Damping resistors of $5 \Omega$ were added in series with the magnet capacitors. To meet the rise time specifications the "front cell" at each PFN entrance was tuned to get a nearly ideal square (fastest) pulse at the magnet input. The fall time was minimised by forcing a zero crossing of the remaining kick component through a mismatch of the terminating magnet resistance (TMR, using a value of $10.7 \Omega$ instead of $10 \Omega$ ) resulting in less post-pulse kick ripple and thus less disturbance to the bunches of the second batch.

\section{Re-commissioning and Kicker Measurements}

After the implementation of the modifications described above the kicker system was re-commissioned in spring 2004 and used during periods of CNGS low intensity extraction, the commissioning of the new LHC transfer line TI 8 and the LHC collimator tests [5-7]. The kick field was measured with capacitive pick-ups (permitting a kick measurement in the SPS) and an inductive probe (as a field calibration reference, not useable in the SPS 


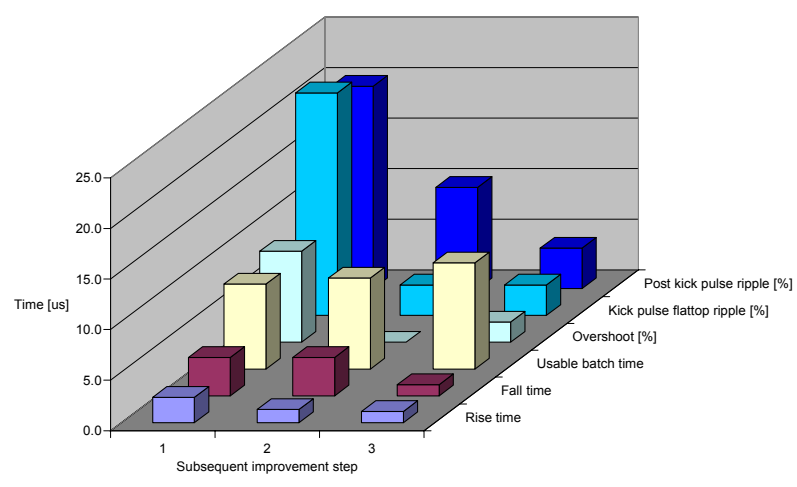

Figure 2: Subsequent improvement steps and resulting MKE pulse parameters; "Step 1" as installed in 2003, "Step 2" after adding magnet damping resistors (laboratory result), "Step 3" after mismatching the TMR values and fine tuning the adjustable PFN front cells.

machine). Kick rise, fall and flattop pulse length times and flattop (overshoot) as well as post-pulse ripple were measured, confirming the effects predicted by the simulations (see Fig. 2).

\section{Further measurements with beam}

After the basic set of parameter measurements additional tests were performed with beam. The kick length was set to the nominal value of $10.5 \mu$ s and the timing of the kick in the beam gap was adjusted by minimising the beam loss monitor (BLMs) signals (see Fig. 3).

To determine the induced betatron oscillation amplitudes the signals from two horizontal (the kicker deflects in the horizontal plane) beam position monitors (BPMs) with a phase advance of about $90^{\circ}$ in between were taken.

A horizontal $1 \sigma$ normalised emittance of $4 \mu \mathrm{m}$ (three times smaller than the design value) and a population of beam tails of about $4 \%$ were measured, using the SPS wire scanners (which were gated at $1 \mu$ s intervals with 1 $\mu$ s lengths). The tails grew to about $11 \%$ when nonlinearities were introduced by powering Landau-damping octupoles to a strength of $-8 \mathrm{~m}^{-4}$. With the SPS transverse feedback damper "off" and the extraction kicker "on" the horizontal emittance grew to $5 \mu \mathrm{m}$ and the population of beam tails to $20 \%$.

\section{Effect of the Transverse Damper}

The CNGS requirements limit the extraction kicker ripple to $\pm 1.5 \%$ [8]. With the nominal extraction kick angle of $0.6 \mathrm{mrad}$ this translates into a betatron oscillation amplitude at the BPMs of $\mathrm{A}_{\text {pu,threshold }}=0.8 \mathrm{~mm}$ [9].

At the nominal CNGS extraction energy of $400 \mathrm{GeV}$, with the transverse feedback "off", betatron oscillation amplitudes did not grow. Once the kick was applied betatron oscillations were excited with growing amplitudes, largest at the beginning and at the end of the batch as bunches located there were affected by the finite rise and fall time of the extraction kick.

When the transverse feedback was activated the amplitudes decreased. Within 140 turns the oscillations in the batch centre were completely damped down. Fig. 4 shows a mountain range plot of the betatron oscillation amplitudes (indicated in red are bucket positions outside the nominal batch length of 2100 bunch positions, for which the intensity exceeded $8 \%$ of the average bucket intensity in the batch core).

Damping of bunches at the edges of the batch was observed to be slow, and not visible on this time scale. Successful damping of these bunches was shown for half of the full time range between two extraction kicks [8]. To study the sensitivity to the extraction kicker timing the timing was shifted by $\pm 50 \mathrm{~ns}$ with respect to the optimum setting indicated in Fig. 3, but even then the oscillations continued to be well damped.

\section{RESULTS}

The results obtained in the 2004 measurement campaign are summarised in Fig. 5 and Table 1, and compared to the data obtained in 2003, before the hardware modifications.



Figure 3: Measured relative SPS beam loss as a function of the extraction kick timing.



Figure 4: Damped betatron oscillation amplitude with the transverse feedback in operation (beam intensity $2.34 \cdot 10^{13}$ protons/batch). 



Figure 5: Measured kick pulse before (2003, red curves) and after hardware modifications (2004, black curves).

Blue dots: $99 \%$ and $4 \%$ kick amplitudes. Note (right hand figure): For comparison the 2003 signal is advanced by $8 \mu$ s.

Table 1: Measured extraction kick pulse parameters

\begin{tabular}{|c|c|c|}
\hline & 2003 & 2004 \\
\hline Rise time $1-99 \%[\mu \mathrm{s}]$ & 2.5 & 1.1 \\
\hline Fall time $99-4 \%[\mu \mathrm{s}]$ & 3.8 & 1.1 \\
\hline Usable batch length $[\mu \mathrm{s}]$ & $2 \cdot 8.4$ & $2 \cdot 10.5^{*}$ \\
\hline |"Kick" pulse flattop ripple| (overshoot) & $11 \%$ & $<3 \%$ \\
\hline Post "kick" pulse ripple & $<20 \%$ & $<4 \%$ \\
\hline
\end{tabular}

\section{SUMMARY}

Since the initial installation of the fast extraction kicker system in 2003 a number of hardware modifications have been applied to improve significantly the performance of this 30-year-old hardware. Various diagnostics added to the original equipment have proven invaluable in this process. Fully matching the new, challenging requirements has nevertheless turned out to be difficult. In the strive to overcome the restrictions it has been successfully demonstrated that the SPS transverse feedback system can be used to damp the ripple induced on the second batch in between the two subsequent extractions (within $50 \mathrm{~ms}=2167 \mathrm{SPS}$ turns) for a linear machine. In conjunction with the damper the fast extraction in LSS4 now fulfils the specification for CNGS double batch extraction, ready for start up of the facility in 2006. This is even the case when the extraction kick timing is shifted by $\pm 50 \mathrm{~ns}$, providing some margin for operation and also for a potential future intensity increase.

\section{REFERENCES}

[1] P.Collier et al., "The SPS as Injector for LHC Conceptual Design", CERN SL 97-07 (DI), 1997.

[2] K.Elsener (Ed.), "General Description of the CERN Project for a Neutrino Beam to Gran Sasso (CNGS)", CERN AC Note 2000-03, December 2000.

[3] M.Benedikt, P.Collier, V.Mertens, J.Poole, K.Schindl (Eds.), "LHC Design Report", Vol. III, The LHC Injector Chain CERN-2004-003, 15 December 2004, pp.159-170.

[4] W.Höfle, E.Vogel, "Simulation of Transient Effects of Beam-Transverse Feedback Interaction with Application to the Extraction of the CNGS Beam from the SPS", CERN Report No. CERN-AB-2005010, January 2005.

[5] E.Shaposhnikova et al., "Recent Intensity Increase in the CERN Accelerator Chain", PAC05, Knoxville, May 2005.

[6] J.Uythoven et al., "Commissioning of the LHC Beam Transfer Line TI 8", PAC05, Knoxville, May 2005.

[7] R.Assmann et al., "LHC Collimation:. Design and Results from Prototyping and Beam Tests", PAC05, Knoxville, May 2005.

[8] M.Meddahi, Twenty-first APC Meeting, CERN, January 2004

[9] E.Gaxiola, W.Höfle, E.Vogel, "Damping of Betatron Oscillations in the SPS Appearing at the Two Step Extraction of CNGS Beam", CERN-AB2005-016-ABP, Geneva, Switzerland, March 2005. 